\title{
Type-Based Analysis of Deadlock for a Concurrent Calculus with Interrupts
}

\author{
Kohei Suenaga ${ }^{1}$ and Naoki Kobayashi ${ }^{2}$ \\ ${ }^{1}$ University of Tokyo \\ 2 Tohoku University
}

\begin{abstract}
The goal of our research project is to establish a type-based method for verification of certain critical properties (such as deadlockand race-freedom) of operating system kernels. As operating system kernels make heavy use of threads and interrupts, it is important that the method can properly deal with both of the two features. As a first step towards the goal, we formalize a concurrent calculus equipped with primitives for threads and interrupts handling. We also propose a type system that guarantees deadlock-freedom in the presence of interrupts. To our knowledge, ours is the first type system for deadlock-freedom that can deal with both thread and interrupt primitives.
\end{abstract}

\section{Introduction}

The goal of our research project is to establish a type-based method for verification of certain critical properties (such as deadlock- and race-freedom) of operating system kernels. As operating system kernels make heavy use of threads and interrupts, it is important that the method can properly deal with both of the two features. Though several calculi that deal with either interrupts [3] or concurrency [1213] have been proposed, none of them deal with both.

Combination of those two features can actually cause errors which are very difficult to find manually. For example, consider the program in Figure 1, The example is taken from an implementation of a protocol stack used in an ongoing research project on cluster computing [11. Though the original source code is written in $\mathrm{C}$, the example is shown in an ML-style language. The function flush_buffer flushes the local buffer and sends pending packets to appropriate destinations. The function receive_data is called when a packet arrives. That function works as an interrupt handler (as specified in the main expression) and is asynchronously called whenever a packet arrives. Since receive_data calls flush_buffer in order for the local buffer to be flushed as soon as the function knows there is a room in the remote buffer (a similar mechanism called congestion control is used in TCP), the following control flow causes deadlock:

$$
\begin{aligned}
\text { Call to flush_buffer } & \rightarrow \text { lock(devlock) } \\
& \rightarrow \text { an interrupt (call to receive_data) } \\
& \rightarrow \text { call to flush_buffer } \rightarrow \text { lock(devlock) }
\end{aligned}
$$




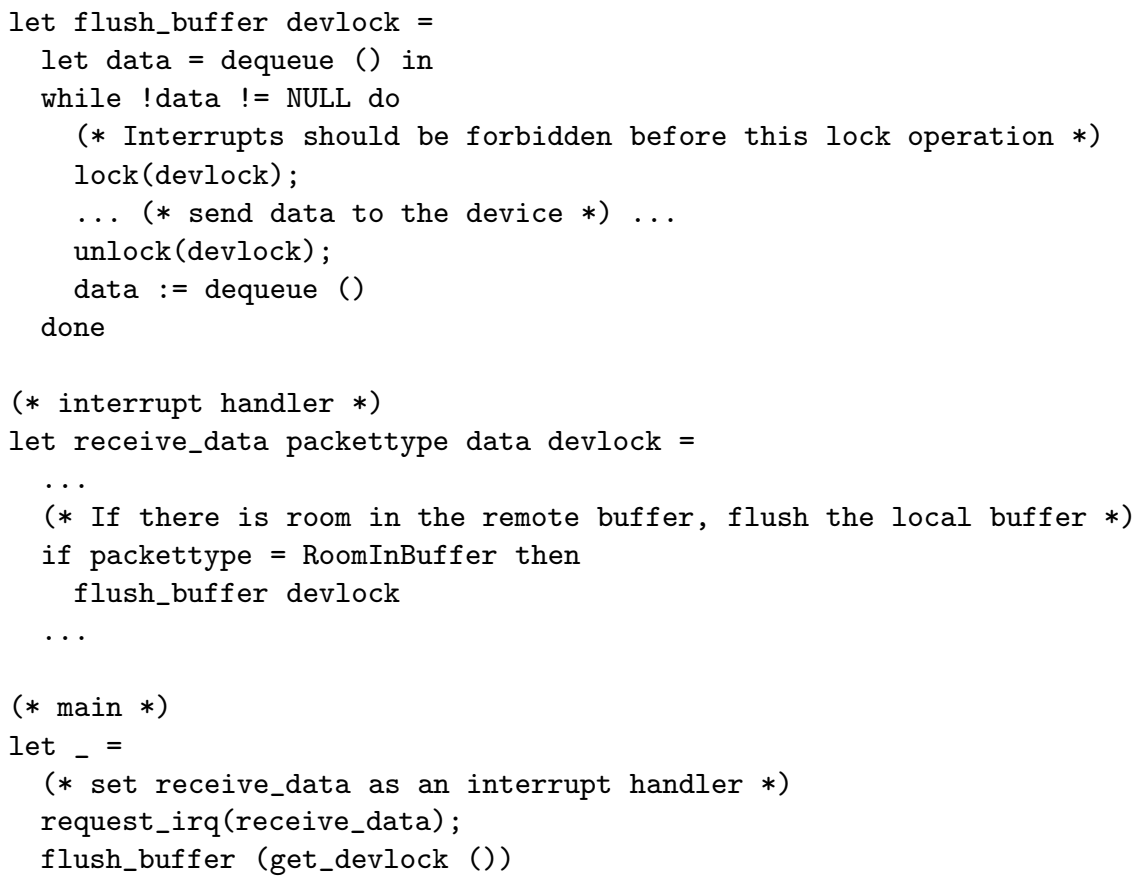

Fig. 1. An example of program which cause deadlock

Note that an interrupt handler does not voluntarily yield. To prevent the deadlock, flush_buffer has to forbid interrupts before it acquires the device lock as shown in Figure 2 .

In order to statically detect such a deadlock, we propose (1) a calculus which is equipped with both interrupts and concurrency and (2) a type system for verifying deadlock-freedom. To our knowledge, ours is the first type system for deadlock-freedom that can deal with both thread and interrupt primitives.

Our type system associates a totally-ordered lock level to each lock and guarantees that locks are acquired in an increasing order of the levels even if interrupts occur. To achieve this, the type system tracks (1) a lower bound of the levels of locks acquired during evaluation and (2) an upper bound of the levels of locks acquired while interrupts are enabled. With our type system, the example in Figure 1 is rejected. On the other hand, if flush_buffer forbids interrupts before it acquires the device lock (as in Figure 2), our type system accepts the program.

The outline of this paper is as follows. Section 2 introduces the syntax and the semantics of our calculus. Section 3 shows our type system and states the type soundness theorem. After discussing related work in Section 4, we conclude in Section 5 . 


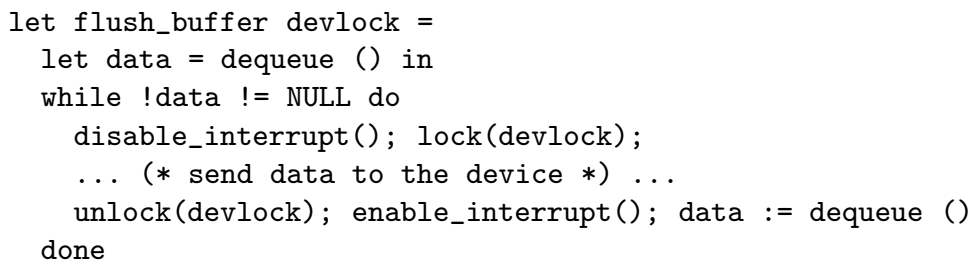

Fig. 2. A correct version of flush_buffer

\section{Target Language}

\subsection{Syntax}

The syntax of our target language is defined in Figure 3. Our language is an imperative language which is equipped with concurrency and interrupt handling.

A program $P$ consists of a sequence of function definitions $\widetilde{D}$ and a main expression $M$. A function definition is constructed from a function name $x$, a sequence of formal arguments $\widetilde{y}$ and a function body. Function definitions can be mutually recursive. Note that a function name belongs to the class of variables, so that one can use a function name as a first-class value.

Expressions are ranged over by a meta-variable $M . \triangleright$ and $\iota$ are left-associative. For the sake of simplicity, we have only block-structured primitives (sync $x$ in $M$ and disable_int $M$ ) for acquiring/releasing locks and disabling/enabling interrupts. We explain intuition of several non-standard primitives below.

$$
\begin{aligned}
x, y, z, f \ldots \in & \operatorname{Var} \\
l c k::= & \text { acquired } \mid \text { released } \\
P::= & \widetilde{D} M \\
D::= & x(\widetilde{y})=M \\
M::= & ()|n| x \mid \text { true } \mid \text { false } \\
& x(\widetilde{v}) \mid \text { let } x=M_{1} \text { in } M_{2} \mid \text { if } v \text { then } M_{1} \text { else } M_{2} \\
\mid & \text { let } x=\text { ref } v \text { in } M|x:=v| ! v \\
& \left(M_{1} \mid M_{2}\right) \mid \text { let } x=\text { newlock }() \text { in } M \\
\mid & \text { sync } x \text { in } M \mid \text { in_sync } x \text { in } M \\
\mid & M_{1} \triangleright M_{2}\left|M_{1} \Psi_{M}\right| \text { disable_int } M \mid \text { in_disable_int } M \\
v::= & () \mid \text { true } \mid \text { false }|n| x \\
E::= & {[] \mid \text { let } x=E \text { in } M } \\
\mid & (E \mid M) \mid(M \mid E) \\
\mid & \text { in_sync } x \text { in } E \mid \text { in_disable_int } E \\
\mid & E \triangleright M \mid M_{1}{ }_{M} E \\
I::= & \text { enabled } \mid \text { disabled }
\end{aligned}
$$

Fig. 3. The Syntax of Our Language 


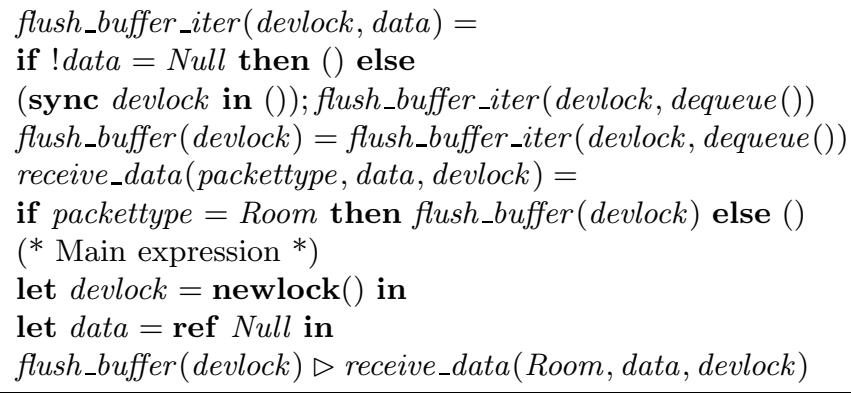

Fig. 4. An Encoding of the Program in Figure 1

- let $x=\operatorname{ref} v$ in $M$ creates a fresh reference to $v$, binds $x$ to the reference and evaluates $M$.

- $M_{1} \mid M_{2}$ is concurrent evaluation of $M_{1}$ and $M_{2}$. Both of $M_{1}$ and $M_{2}$ should evaluate to ().

- let $x=$ newlock ( $)$ in $M$ generates a new lock, binds $x$ to the lock and evaluates $M$.

- sync $x$ in $M$ attempts to acquire the lock $x$ and evaluates $M$ after the lock is acquired. After $M$ is evaluated to a value, the lock $x$ is released.

- $M_{1} \triangleright M_{2}$ installs an interrupt handler $M_{2}$ and evaluates $M_{1}$. Once an interrupt occurs, $M_{1}$ is suspended until $M_{2}$ evaluates to a value. When $M_{1}$ evaluates to a value $v, M_{1} \triangleright M_{2}$ evaluates to $v$.

- disable_int $M$ disables interrupts during an evaluation of $M$.

The following three primitives only occur during evaluation and should not be included in programs.

- in_sync $x$ in $M$ represents the state in which $M$ is being evaluated with the lock $x$ acquired. After $M$ evaluates to a value, the lock $x$ is released.

- $M_{1} \boldsymbol{\varangle}_{M} M_{2}$ represents the state in which the interrupt handler $M_{2}$ is being evaluated. After $M_{2}$ evaluates to a value, the interrupted expression $M_{1}$ and the initial state of interrupt handler $M$ are recovered.

- in_disable_int $M$ represents the state in which $M$ is being evaluated with interrupts disabled. After $M$ evaluates to a value, interrupts are enabled.

We write $M_{1} ; M_{2}$ for let $x=M_{1}$ in $M_{2}$ where $x$ is not free in $M_{2}$.

Figure 4 shows how the example in Figure 1 is encoded in our language. Though that encoding does not strictly conform to the syntax of our language (e.g., flush_buffer_iter is applied to an expression dequeue(), not to a value), one can easily translate the program into one that respects our syntax.

Our interrupt calculus is very expressive and can model various interrupt mechanisms, as discussed in Examples 14 below.

Example 1. In various kinds of CPUs, there is a priority among interrupts. In such a situation, if an interrupt with a higher priority occurs, interrupts with 
lower priorities do not occur. We can express such priorities by connecting several expressions with $\triangleright$ as follows.

$$
\text { do_something }(\ldots) \triangleright \text { interrupt_low }(\ldots) \triangleright \text { interrupt_high }(. .)
$$

If an interrupt occurs in do_something (...) $\triangleright$ interrupt_low (...) (note that $\triangleright$ is left-associative), the example above is reduced to

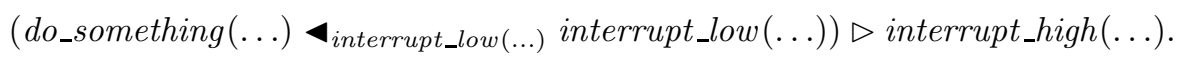

That state represents that interrupt_low interrupted do_something. From that state, interrupt_high can still interrupt.

$$
\begin{aligned}
& \left(\text { do_something }(\ldots) \boldsymbol{\triangleleft}_{\text {interrupt_low }(\ldots)} \text { interrupt_low }(\ldots)\right)
\end{aligned}
$$

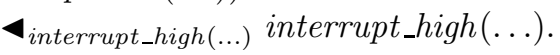

interrupt_high can interrupt also from the initial state.

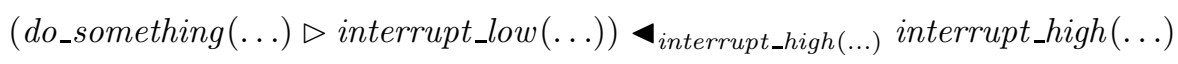

From the state above, interrupt_low cannot interrupt until interrupt_high(...) evaluates to a value.

Example 2. In our calculus, we can locally install interrupt handlers. Thus, we can express a multi-threaded program in which an interrupt handler is installed on each thread.

$$
(\text { thread1 }(\ldots) \triangleright \text { handler1 }(\ldots)) \mid(\text { thread2 }(\ldots) \triangleright \text { handler2 }(\ldots)) \ldots
$$

This feature is useful for modeling a multi-CPU system in which even if an interrupt occurs in one $\mathrm{CPU}$, the other CPUs continue to work in non-interrupt mode.

Example 3. In the example in Figure 4, we assume that no interrupt occur in the body of receive_data. One can express that an interrupt may occur during an execution of receive_data by re-installing an interrupt handler as follows.

receive_data $($ packettype, data, devlock $)=$

(if packettype $=$ Room then flush_buffer (devlock) else ()) $\triangleright$ receive_data $($ Room, data, devlock $)$

Example 4. Since many operating system kernels are written in C, we make design decisions of our language based on that of $\mathrm{C}$. For example, names of functions are first-class values in our language because $\mathrm{C}$ allows one to use a function name as a function pointer and because operating system kernels heavily use function pointers. With this feature, we can express a runtime change of interrupt handler as follows:

$$
\text { let } x=\operatorname{ref} f \text { in }((\ldots ; x:=g ; \ldots) \triangleright(! x)())
$$

Until $g$ is assigned to the reference $x$, the installed interrupt handler is $f$. After the assignment, the interrupt handler is $g$. This characteristic is useful for modeling operating system kernels in which interrupt handlers are changed when, for example, device drivers are installed. 


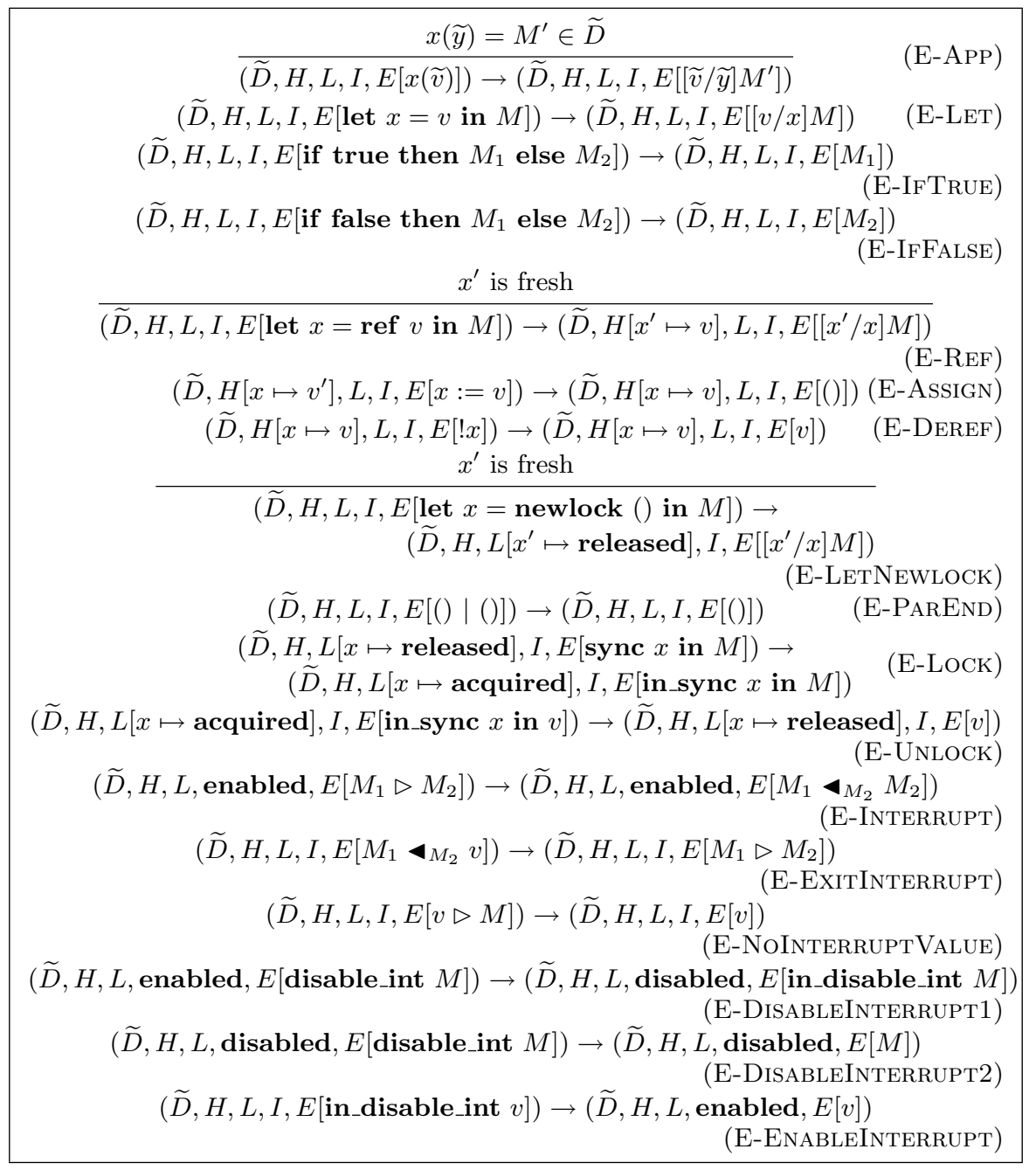

Fig. 5. The Operational Semantics of Our Language

\subsection{Operational Semantics}

The semantics is defined as rewriting of a configuration $(\widetilde{D}, H, L, I, M) . H$ is a heap, which is a map from variables to values. (Note that references are represented by variables.) $L$ is a map from variables to $\{$ acquired, released $\} . I$ is an interrupt flag, which is either enabled or disabled 11

\footnotetext{
${ }^{1}$ We do not assign an interrupt flag to each interrupt handler in order to keep the semantics simple. Even if we do so, the type system introduced in Section 3 can be used with only small changes.
} 
Figure 5 shows the operational semantics of our language. We explain several important rules.

- In (E-ReF) and (E-LETNEwLock), newly generated references and locks are represented by fresh variables.

- Reduction with the rule (E-LOCK) succeeds only if the lock being acquired is not held. (E-UNLOCK) is similar.

- disable_int changes the interrupt flag only when the flag was enabled (rule (E-DisABLEINTERRUPT1)). Otherwise, disable_int does nothing (rule (E-DisABleINTERRUPT2)).

- If the interrupt flag is enabled, then a handler $M_{2}$ can interrupt $M_{1}$ anytime with the rule (E-INTERRUPT). When the interrupt occurs, the initial expression of interrupt handler $M_{2}$ is saved. After the handler terminates, the saved expression is recovered with (E-ExITINTERRUPT).

The following example shows how the program in Figure 4 leads to a deadlocked state. We write $L_{u}$ for $\left\{\right.$ devlock $^{\prime} \mapsto$ released $\}$ and $L_{l}$ for $\left\{\right.$ devlock ${ }^{\prime} \mapsto$ acquired $\}$. We omit $\widetilde{D}, H$ and $I$ components of configurations.

$\left(L_{u}\right.$, flush_buffer $\left(\right.$ devlock $\left.^{\prime}\right) \triangleright$ receive_data $\left(\right.$ Room, data, devlock $\left.\left.{ }^{\prime}\right)\right)$

$\rightarrow^{*}\left(L_{u}\right.$, sync devlock' in ()$\triangleright$ receive_data $($ Room, data, devlock $\left.)\right)$

$\rightarrow\left(L_{l}\right.$, in_sync devlock ${ }^{\prime}$ in ()$\triangleright$ receive_data $($ Room, data, devlock $\left.)\right)$

$\rightarrow\left(L_{l}\right.$, in_sync devlock ${ }^{\prime}$ in ()$\triangleleft_{\text {receive_data }(\ldots)}$ receive_data $($ Room, data, devlock $\left.)\right)$

$\rightarrow^{*}\left(L_{l}\right.$, in_sync devlock $k^{\prime}$ in () $\boldsymbol{\triangleleft}_{\text {receive_data }(. . .)}$ flush_buffer $\left(\right.$ devlock $\left.\left.k^{\prime}\right)\right)$

$\rightarrow^{*}\left(L_{l}\right.$, in_sync devlock ${ }^{\prime}$ in ()$\varangle_{\text {receive_data }(\ldots)}$ sync devlock ${ }^{\prime}$ in ()$)$

The last configuration is in a deadlock because the attempt to acquire devlock', which is already acquired in $L_{l}$, never succeeds and because the interrupt handler sync devlock' in () does not voluntarily yield.

\section{Type System}

\subsection{Lock Levels}

In our type system, every lock type is associated with a lock level, which is represented by a meta-variable lev. The set of lock levels is $\{-\infty, \infty\} \cup \mathbb{N}$, where $\mathbb{N}$ is the set of natural numbers. We extend the standard partial order $\leq$ on $\mathbb{N}$ to that on $\{-\infty, \infty\} \cup \mathbb{N}$ by $\forall$ lev $\in\{-\infty, \infty\} \cup \mathbb{N}$. $-\infty \leq$ lev $\leq \infty$. We write $l e v_{1}<l e v_{2}$ for $l e v_{1} \leq l e v_{2} \wedge l e v_{1} \neq l e v_{2}$.

\subsection{Effects}

Our type system guarantees that a program acquires locks in a strict increasing order of lock levels. To achieve this, we introduce effects which describe how a program acquires locks during evaluation.

An effect, represented by a meta-variable $\varphi$, is a pair of lock levels $\left(\operatorname{lev}_{1}, \operatorname{lev}_{2}\right)$. The meaning of each component is as follows. 


$$
\begin{aligned}
\tau & ::=\text { unit } \mid \text { int } \mid \text { bool }\left|\widetilde{\tau}_{1} \stackrel{\varphi}{\rightarrow} \tau_{2}\right| \tau \text { ref } \mid \operatorname{lock}(\text { lev }) \\
l e v & \in\{-\infty, \infty\} \cup \mathbb{N} \\
\varphi & ::=\left(\text { lev }_{1}, \text { lev }_{2}\right)
\end{aligned}
$$

Fig. 6. Syntax of types

- $l e v_{1}$ is a lower bound of the lock levels of locks that may be acquired.

- $l e v_{2}$ is an upper bound of the lock levels of locks that may be acquired or have been acquired while interrupts are enabled.

For example, an effect $(0,-\infty)$ means that locks whose levels are more than or equal to 0 may be acquired and that no locks are acquired while interrupts are enabled. An effect $(0,1)$ means that locks whose levels are more than or equal to 0 may be acquired and that a lock of level 1 may be acquired or has already been acquired while interrupts are enabled. We write $\emptyset$ for $(\infty,-\infty)$.

We define the subeffect relation and the join operator for effects as follows.

Definition 1 (Subeffect Relation). (lev $\left.1, l e v_{2}\right) \leq\left(l e v_{1}^{\prime}, l e v_{2}^{\prime}\right)$ holds if and only if $l e v_{1}^{\prime} \leq l e v_{1}$ and $l e v_{2} \leq l e v_{2}^{\prime}$.

$\left(l e v_{1}, l e v_{2}\right) \leq\left(l e v_{1}^{\prime}, l e v_{2}^{\prime}\right)$ means that an expression that acquires locks according to the effect $\left(l e v_{1}, l e v_{2}\right)$ can be seen as an expression with the effect $\left(l e v_{1}^{\prime}, l e v_{2}^{\prime}\right)$. For example, $(1,2) \leq(0,3)$ holds. $\emptyset$ is the bottom of $\leq$.

Definition 2 (Join). $\left(l e v_{1}, l e v_{2}\right) \sqcup\left(l e v_{1}^{\prime}, \operatorname{lev}_{2}^{\prime}\right)=\left(\min \left(l e v_{1}, l e v_{1}^{\prime}\right), \max \left(l e v_{2}, l e v_{2}^{\prime}\right)\right)$ For example, $(1,2) \sqcup(0,1)=(0,2)$ and $(0,-\infty) \sqcup(1,2)=(0,2)$ hold. $\emptyset$ is an identity of $\sqcup$.

\subsection{Syntax of Types}

Figure 6 shows the syntax of types and effects. A type, represented by a metavariable $\tau$, is either unit, int, bool, $\widetilde{\tau}_{1} \stackrel{\varphi}{\rightarrow} \tau_{2}, \tau$ ref or $\operatorname{lock}(l e v)$. We write $\widetilde{\tau}$ for a sequence of types. $\tau$ ref is the type of a reference to a value of type $\tau$. $\widetilde{\tau}_{1} \stackrel{\varphi}{\rightarrow} \tau_{2}$ is the type of functions which take a tuple of values of type $\widetilde{\tau}_{1}$ and return a value of type $\tau_{2} \cdot \varphi$ is the latent effect of the functions.

\subsection{Type Judgment}

The type judgment form of our type system is $\Gamma \vdash M: \tau \& \varphi$ where $\Gamma$ is a map from variables to types. The judgment means that the resulting value of the evaluation of $M$ has type $\tau$ if an evaluation of $M$ under an environment described by $\Gamma$ terminates, and that locks are acquired in a strict increasing order of lock levels during the evaluation. The minimum and maximum lock levels acquired are constrained by $\varphi$. For example, $x: \operatorname{lock}(0), y: \operatorname{lock}(1) \vdash$ sync $x$ in sync $y$ in () : unit \& $(0,1)$ and $x: \operatorname{lock}(0), y: \operatorname{lock}(1) \vdash$ sync $x$ in (disable_int sync $y$ in ()$)$ : unit \& $(0,0)$ hold. 
Definition 3. The relation $\Gamma \vdash M: \tau \& \varphi$ is the smallest relation closed under the rules in Figures 7 and 8 . The predicate noIntermediate $(M)$ in Figure 8 holds if and only if $M$ does not contain in_sync $x$ in $M^{\prime}$, in_disable_int $M^{\prime}$ or $M_{1} \boldsymbol{\sim}_{M^{\prime}} M_{2}$ as subterms.

We explain several important rules.

- (T-Sync): If the level of $x$ is lev, then $M$ can acquire only locks whose levels are more than lev. That is guaranteed by the condition lev $<l e v_{1}$ where $l e v_{1}$ is a lower bound of the levels of locks that may be acquired by $M$.

- (T-DisableInterrupt): The second component of the effect of disable_int $M$ is changed to $-\infty$ because no interrupt occurs in $M$, so that no locks are acquired by interrupt handlers.

- (T-InstHandLER): The second component of the effect of $M_{1}$ should be less than the first component of the effect of $M_{2}$ because $M_{2}$ can interrupt $M_{1}$ at any time. This is why we need to include the maximum level in effects.

- (T-FundeF): The condition $\varphi^{\prime} \leq \varphi_{i}$ guarantees that the latent effect of the type of the function being defined soundly approximates the runtime locking behavior.

We show how the program in Figure 4 is rejected in our type system. From the derivation tree in Figure 9, flush_buffer_iter has a type $\left(\operatorname{lock}(1), \tau_{d}\right.$ ref $) \stackrel{(1,1)}{\longrightarrow}$ unit, where $\tau_{d}$ is the type of the contents of the reference data. Thus, flush_buffer has a type $\operatorname{lock}(1) \stackrel{(1,1)}{\longrightarrow}$ unit and receive_data has a type $\left(\tau_{p}, \tau_{d}, \operatorname{lock}(1)\right) \stackrel{(1,1)}{\longrightarrow}$ unit, where $\tau_{p}$ is the type of packettype.

Consider the main expression of the example. Let $\Gamma$ be devlock : $\operatorname{lock}(1)$, data: $\tau_{d}$ ref. Then, we have

- $\Gamma \vdash$ flush_buffer(devlock) : unit \& $(1,1)$ and

- $\Gamma \vdash$ receive_data $($ Room, data, devlock $)$ : unit \& $(1,1)$.

However, the condition $l e v_{2}<l e v_{1}^{\prime}$ of the rule (T-INSTHANDLER) prevents the main expression to be well-typed $(1<1$ does not hold).

Suppose that sync devlock in () in the body of flush_buffer_iter is replaced by disable_int sync devlock in (). Then, flush_buffer_iter has a type $\left(\operatorname{lock}(1), \tau_{d}\right.$ ref $) \stackrel{(1,-\infty)}{\rightarrow}$ unit Thus, because $\Gamma \vdash$ flush_buffer (devlock) : unit $\&(1,-\infty)$ and $-\infty<1$ hold, the program is well-typed.

\subsection{Type Soundness}

We prove the soundness of our type system. Here, type soundness means that a well-typed program does not get deadlocked if one begins an evaluation of the program under an initial configuration (i.e., under an empty heap, an empty lock environment and enabled interrupt flag).

We first define deadlock. The predicate deadlocked $(L, M)$ defined below means that $M$ is in a deadlocked state under $L$. 


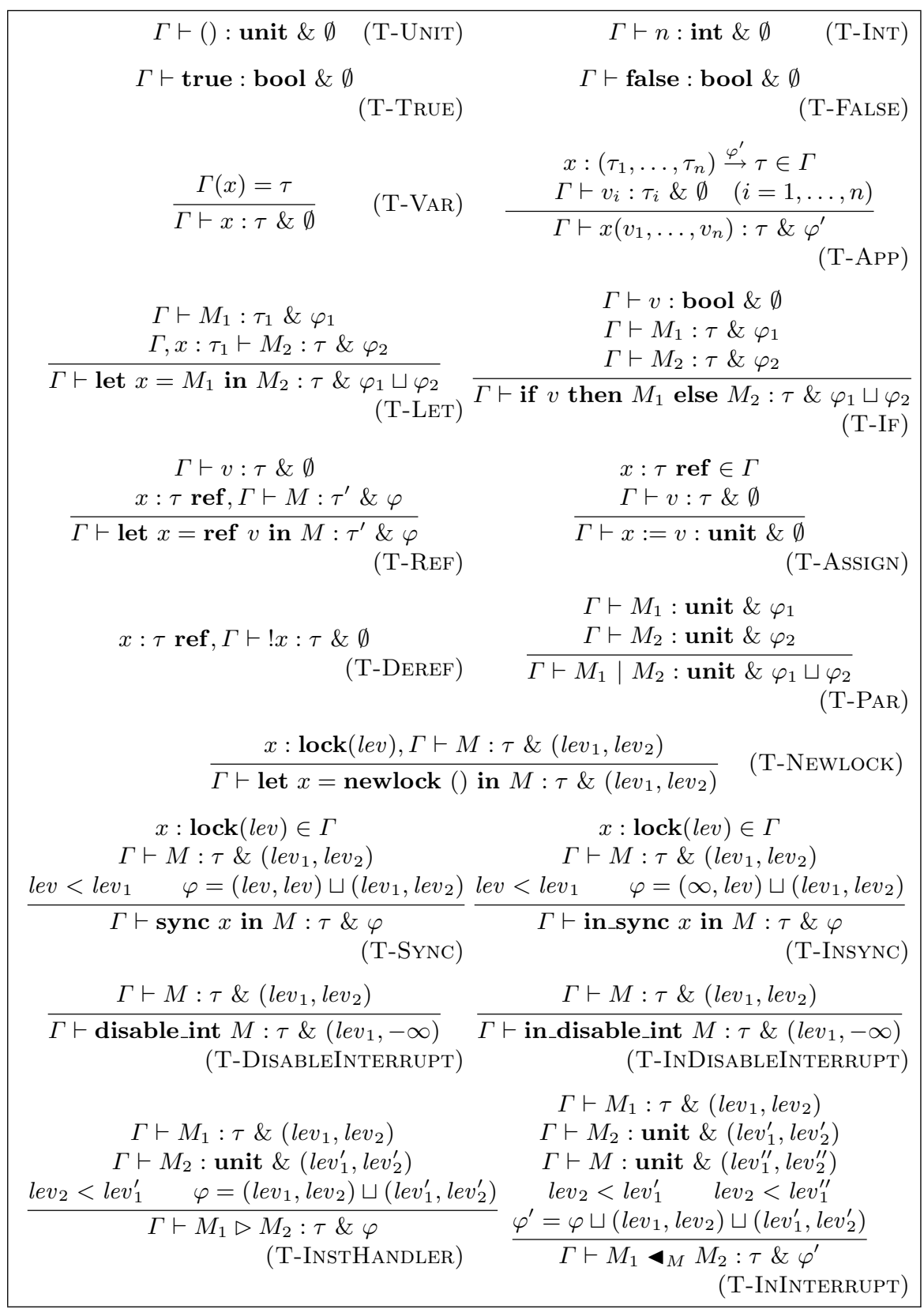

Fig. 7. Typing rules

Definition 4 (Deadlock). The predicate deadlocked $(L, M)$ holds if and only if for all $E$ and $i, M=E[i]$ implies that there exist $x$ and $M^{\prime}$ such that $i=$ sync $x$ in $M^{\prime} \wedge L(x)=$ acquired. Here, $i$ is defined by the following syntax. 


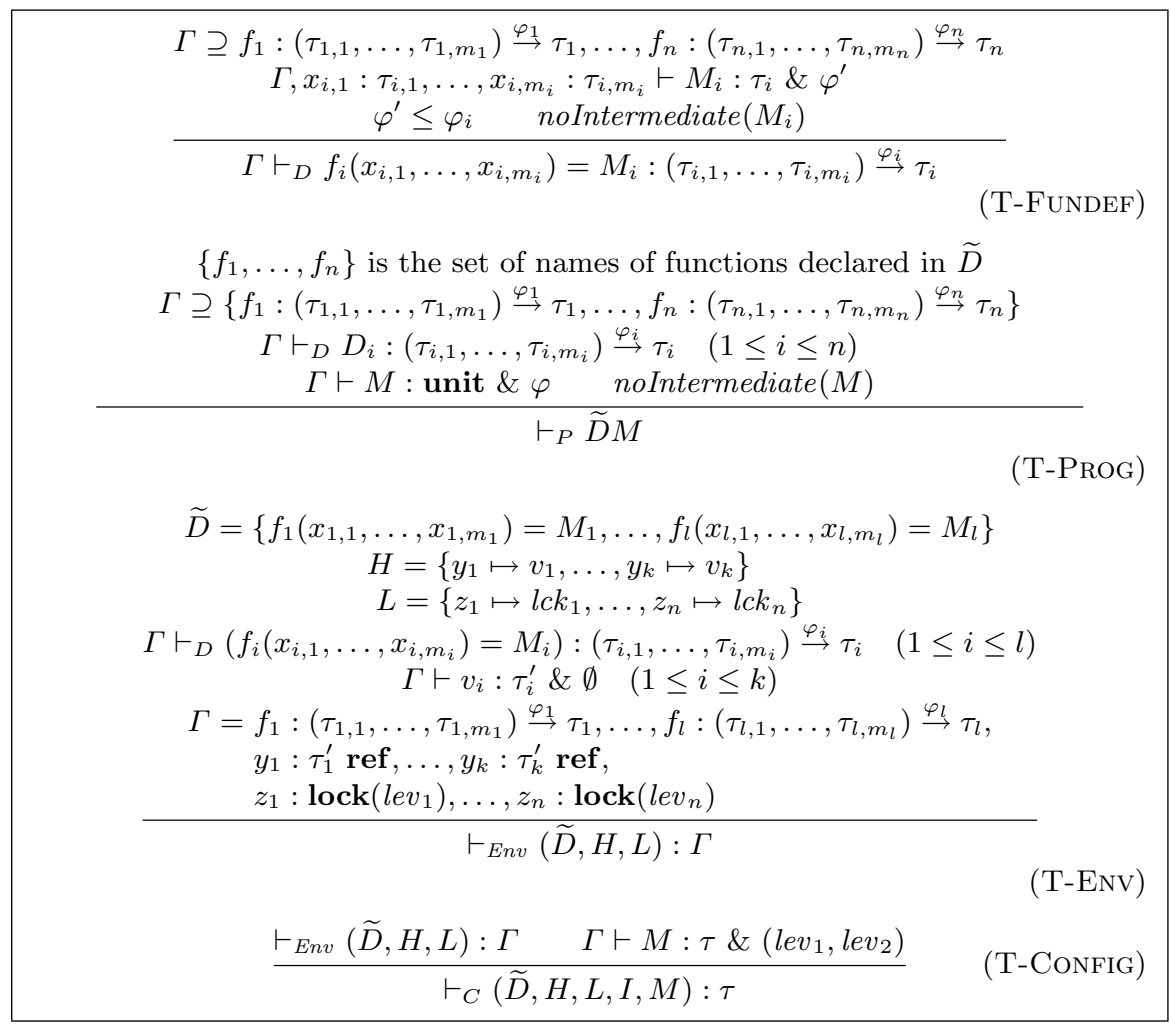

Fig. 8. Typing Rules for Program and Configuration

\section{$i::=x(\widetilde{v}) \mid$ let $x=v$ in $M$}

if true then $M_{1}$ else $M_{2} \mid$ if false then $M_{1}$ else $M_{2}$

let $x=\operatorname{ref} v$ in $M|x:=v| ! x$

let $x=$ newlock () in $M|(() \mid())|$ sync $x$ in $M \mid$ in_sync $x$ in $v$ $M_{1} \varangle_{M_{2}} v|v \triangleright M|$ disable_int $M \mid$ in_disable_int $v$

In the definition above, $i$ is a term that can be reduced by the rules in Figure 5. Thus, deadlocked $(L, M)$ means that every reducible subterm in $M$ is a blocked lock-acquiring instruction. For example,

deadlocked $(L,($ in_sync $x$ in (sync $y$ in 0$)) \mid($ in_sync $y$ in (sync $x$ in 0$)))$

holds where $L=\{x \mapsto$ acquired, $y \mapsto$ acquired $\}$.

Note that $M_{1} \triangleright M_{2}$ is not included in the definition of $i$ because, in the real world, whether $M_{1} \triangleright M_{2}$ is reducible or not depends on the external environment which is not modeled in our calculus. For example, (sync $x$ in ()$) \triangleright()$ is deadlocked under the environment in which $x$ is acquired.

Theorem 1 (Type Soundness). If $\vdash_{P} \widetilde{D} M$ and $(\widetilde{D}, \emptyset, \emptyset$, enabled, $M) \rightarrow^{*}$ $\left(\widetilde{D}^{\prime}, H^{\prime}, L^{\prime}, I^{\prime}, M^{\prime}\right)$, then $\neg$ deadlocked $\left(L^{\prime}, M^{\prime}\right)$. 


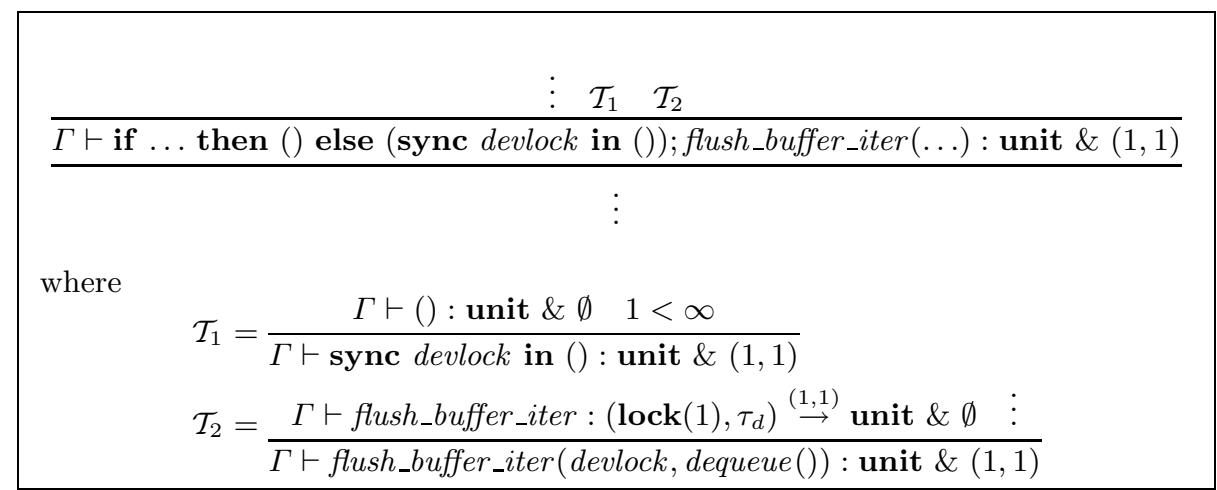

Fig. 9. Derivation Tree of the body of flush_buffer_iter. $\Gamma=$ flush_buffer_iter : $\left(\operatorname{lock}(1), \tau_{d}\right.$ ref $) \stackrel{(1,1)}{\rightarrow}$ unit,flush_buffer $\quad: \quad \operatorname{lock}(1) \stackrel{(1,1)}{\longrightarrow}$ unit, receive_data : $\left(\tau_{p}, \tau_{d} \operatorname{ref}, \operatorname{lock}(1)\right) \stackrel{(1,1)}{\longrightarrow}$ unit, devlock $: \operatorname{lock}(1)$, data $: \tau_{d}$.

The theorem above follows from Lemmas 14 below. In those lemmas, we use a predicate wellformed $(L, I, M)$ which means that $L, I$ and the shape of $M$ are consistent.

Definition 5. wellformed $(L, I, M)$ holds if and only if

- $L(x)=$ released or $x \notin \operatorname{Dom}(L)$ implies that $M$ does not contain in_sync $x$,

- $L(x)=$ acquired implies $\operatorname{AckIn}(x, M)$,

- $I=$ enabled implies that $M$ does not contain in_disable_int.

$-I=$ disabled implies that there exist $E$ and $M^{\prime}$ such that $M=$ $E\left[\right.$ in_disable_int $\left.M^{\prime}\right]$ and both $E$ and $M^{\prime}$ do not contain in_disable_int.

Here, $\operatorname{AckIn}(x, M)$ is the least predicate that satisfies the following rules.

\begin{tabular}{|c|c|}
\hline$\frac{E \text { and } M^{\prime} \text { do not contain in_sync } x \text { in }}{\left.A c k \ln \left(x \text { in svnc } x \text { in } M^{\prime}\right]\right)}$ & $\begin{array}{l}\operatorname{AckIn}\left(x, M_{1}\right) \\
E, M^{\prime} \text { and } M_{2} \text { do not contain } \\
\text { in_sync } x \text { in }\end{array}$ \\
\hline (ACKIN-BASE) & $\begin{aligned} \operatorname{AckIn}\left(x, E\left[M_{1} \boldsymbol{\Psi}_{M^{\prime}} M_{2}\right]\right) \\
(\mathrm{ACKIN-INTERRUPT})\end{aligned}$ \\
\hline
\end{tabular}

Lemma 1. If $\vdash_{P} \widetilde{D} M$, then wellformed $(\emptyset$, enabled, $M)$ and $\vdash_{C}(D, \emptyset, \emptyset$, enabled, $M)$.

Lemma 2. If wellformed $(L, I, M)$ and $(\widetilde{D}, H, L, I, M) \rightarrow\left(\widetilde{D}^{\prime}, H^{\prime}, L^{\prime}, I^{\prime}, M^{\prime}\right)$, then wellformed $\left(L^{\prime}, I^{\prime}, M^{\prime}\right)$.

Lemma 3 (Preservation). If $\vdash_{C}(\widetilde{D}, H, L, I, M): \tau$ and $(\widetilde{D}, H, L, I, M) \rightarrow$ $\left(\widetilde{D}^{\prime}, H^{\prime}, L^{\prime}, I^{\prime}, M^{\prime}\right)$, then $\vdash_{C}\left(\widetilde{D}^{\prime}, H^{\prime}, L^{\prime}, I^{\prime}, M^{\prime}\right): \tau$.

Lemma 4 (Deadlock-Freedom). If $\vdash_{C}(\widetilde{D}, H, L, I, M): \tau$ and wellformed $(L, I, M)$, then $\neg$ deadlocked $(L, M)$.

Proofs of those lemmas are in the full version of this paper. 


\subsection{Type Inference}

We can construct a standard constraint-based type inference algorithm as follows. The algorithm takes a program as an input, prepares variables for unknown types and lock levels, and extracts constraints on them based on the typing rules. By the standard unification algorithm and the definition of the subeffect relation, the extracted constraints can then be reduced to a set of constraints of the form $\left\{\rho_{1} \geq \xi_{1}, \ldots, \rho_{n} \geq \xi_{n}\right\}$ where the grammar for $\xi_{1}, \ldots, \xi_{n}$ is given by

$$
\begin{aligned}
& \xi::=\rho \text { (lock level variables) } \\
& \quad|\quad-\infty| \infty\left|\min \left(\xi_{1}, \xi_{2}\right)\right| \max \left(\xi_{1}, \xi_{2}\right) \mid \xi+1 .
\end{aligned}
$$

Note that $l e v<l e v_{1}$ in (T-SYNC) can be replaced by lev $+1 \leq l e v_{1}$. The constraints above can be solved as in Kobayashi's type-based deadlock analysis for the $\pi$-calculus [7]. We will formalize the algorithm in the full version of the current paper.

\section{Related Work}

Chatterjee et al. have proposed a calculus that is equipped with interrupts 314. They also proposed a static analysis of stack boundedness (i.e., interrupt chains cannot be infinite) of programs. The main differences between our calculus and their calculus are as follows. (1) Their calculus is not equipped with concurrency primitives. (2) Each handler has its own interrupt flag in their calculus. (3) Our calculus can express an install, a change and a detach of interrupt handlers. Due to (1), we cannot use their calculus to discuss deadlock-freedom analysis. As for (2), their calculus has an interrupt mask register (imr) to control which handlers are allowed to interrupt and which are not. This feature is indispensable in the verification of operating system kernels. We can extend our calculus to incorporate this feature by adding a tag to each interrupt handler $\left(M \triangleright\left\{t_{1}: M_{1}, \ldots, t_{n}: M_{n}\right\}\right)$ and by specifying a tag on interrupt disabling primitives (disable_int $t$ in $M$ ). A handler with tag $t$ cannot interrupt inside disable_int $t$ in. We also extend effects like (lev,taglevel), where taglevel is a map from tags to lock levels. taglevel $(t)$ is an upper bound of the lock levels of locks that may be acquired or have been acquired while interrupts specified by $t$ is enabled. Typing rules need to be modified accordingly. Concerning (3), our calculus can express a change of interrupt handlers as shown in Section 2 .

Much work 22789] on deadlock-freedom analysis of concurrent programs has been done. However, none of them deal with interrupts. Kobayashi et al. [7819] have proposed type systems for deadlock-freedom of $\pi$-calculus processes. Their idea is (1) to express how each channel is used as a usage expression and (2) to add capability levels and obligation levels to the inferred usage expression in order to detect circular dependency among input/output operations to channels. Their capability/obligation levels correspond to our lock levels. Their usage expressions are unnecessary in the present framework because our synchronization primitive is block-structured. That notion would be useful if we allow non-block-structured 
lock primitives. Flanagan and Abadi [14] have proposed a type-based deadlockfreedom and race-freedom analysis for a Java-like language. Though their type system also uses lock levels, they need to track only a lower bound of acquired level as an effect because they do not deal with interrupts. In our type system, we need to track lower and upper bounds of levels as an effect in order to guarantee deadlock-freedom in the presence of interrupts.

Asynchronous exceptions 510 in Java and Haskell are similar to interrupts in that both cause an asynchronous jump to an exception/interrupt handler. Asynchronous exceptions are the exceptions that may be unexpectedly thrown during an execution of a program as a result of some events such as timeouts or stack overflows. Marlow et al. [10] extended Concurrent Haskell [6] with support for handling asynchronous exceptions. However, an asynchronous exception does not require the context in which the exception is thrown to be resumed after an exception handler returns, while an interrupt requires the context to be resumed.

\section{Conclusion}

We have proposed a calculus which is equipped with concurrency and interrupts. We have also proposed a type system for verification of deadlock-freedom for the calculus.

There remain much work to be done to make our framework applicable to verification of real operating system kernels. Since many operating system kernels are written in $\mathrm{C}$, we need to include records, arrays and pointer arithmetics in our calculus. For those extensions, we may also need to refine the type system. In the current lock-level-based approach, a lock level is statically assigned to each syntactic occurence of a lock, so that the same lock level may be assigned to different locks. To prevent that problem, we may need to introduce lock-level polymorphism and run-time ordering of lock levels as proposed in [2].

We also plan to develop type systems for verifying other crucial safety properties such as race-freedom and atomicity.

\section{Acknowledgement}

We are grateful to Eijiro Sumii, Hiroya Matsuba, Toshiyuki Maeda and Yutaka Ishikawa for the comment on this research. We are also grateful to the anonymous reviewers for their fruitful comments.

\section{References}

1. Martín Abadi, Cormac Flanagan, and Stephen N. Freund. Types for safe locking: Static race detection for java. ACM Transactions on Programming Languages and Systems, 28(2):207-255, March 2006. 
2. Chandrasekhar Boyapati, Robert Lee, and Martin Rinard. Ownership types for safe programming: Preventing data races and deadlocks. In Proceedings of the 2002 ACM SIGPLAN Conference on Object-Oriented Programming Systems, Languages and Applications, (OOPSLA 2002), volume 37 of SIGPLAN Notices, pages 211-230, November 2002.

3. Krishnendu Chatterjee, Di Ma, Rupak Majumdar, Tian Zhao, Thomas A. Henzinger, and Jens Palsberg. Stack size analysis for interrupt-driven programs. Information and Computation, 194(2):144-174, 2004.

4. Cormac Flanagan and Martín Abadi. Types for safe locking. In Proceedings of 8the European Symposium on Programming (ESOP'99), volume 1576 of Lecture Notes in Computer Science, pages 91-108, March 1999.

5. James Gosling, Bill Joy, Guy Steele, and Gilad Bracha. The Java Language Specification, Third Edition. Addison-Wesley Professional, June 2005.

6. Simon Peyton Jones, Andrew Gordon, and Sigbjorn Finne. Concurrent haskell. In Proceedings of the 23rd ACM SIGPLAN-SIGACT Symposium on Principles of Programming Languages (POPL 1996), pages 295-308, January 1996.

7. Naoki Kobayashi. Type-based information flow analysis for the pi-calculus. Acta Informatica, 42(4-5):291-347, 2005.

8. Naoki Kobayashi. A new type system for deadlock-free processes. In Proceedings of the 17th International Conference on Concurrency Theory, volume 4137 of Lecture Notes in Computer Science, pages 233-247, August 2006.

9. Naoki Kobayashi, Shin Saito, and Eijiro Sumii. An implicitly-typed deadlock-free process calculus. In Proceedings of CONCUR 2000, volume 1877 of Lecture Notes in Computer Science, pages 489-503, August 2000.

10. Simon Marlow, Simon Peyton Jones, and Andrew Moran. Asynchronous exceptions in haskell. In Proceedings of ACM SIGPLAN 2001 Conference on Programming Language Design and Implementation (PLDI 2001), June 2001.

11. Hiroya Matsuba and Yutaka Ishikawa. Single IP address cluster for internet servers. In Proceedings of 21st IEEE International Parallel and Distributed Processing Symposium (IPDPS2007), March 2007.

12. Robin Milner. Communication and Concurrency. Prentice Hall, September 1995.

13. Robin Milner. Communicating and Mobile Systems: the Pi-Calculus. Cambridge University Press, 1999.

14. Jens Palsberg and Di Ma. A typed interrupt calculus. In Proceedings of 7 th International Symposium on Formal Techniques in Real-Time and Fault Tolerant Systems, volume 2469 of Lecture Notes in Computer Science, pages 291-310, September 2002. 